\title{
Aging and diabetes drive the COVID-19 forwards; unveiling nature and existing therapies for the treatment
}

\author{
Udeep Chawla ${ }^{1} \cdot$ Manoj Kumar Kashyap ${ }^{2} \cdot$ Amjad Husain $^{3,4}$ (D) \\ Received: 4 February 2021 / Accepted: 27 May 2021 / Published online: 24 June 2021 \\ (c) The Author(s), under exclusive licence to Springer Science+Business Media, LLC, part of Springer Nature 2021
}

\begin{abstract}
Human SARS Coronavirus-2 (SARS-CoV-2) has infected more than 170 million people worldwide and resulted in more than 3.5 million deaths so far. The infection causes Coronavirus disease (COVID-19) in people of all age groups, notably diabetic and old age people, at a higher risk of infectivity and fatality. Around 35\% of the patients who have died of the disease were diabetic. The infection is associated with weakening immune response, chronic inflammation, and potential direct pancreatic impairment. There seems to be a three-way association of the SARS-CoV-2 infection with diabetes and aging. The COVID19 infection causes metabolism complications, which may induce diabetes and accelerate aging in healthy individuals. How does diabetes elevate the likelihood of the infection is not clearly understood. we summarize mechanisms of accelerated aging in COVID-19 and diabetes, and the possible correlation of these three diseases. Various drug candidates under different stages of pre-clinical or clinical developments give us hope for the development of COVID-19 therapeutics, but there is no approved drug so far to treat this disease. Here, we explored the potential of anti-diabetic and anti-aging natural compounds for the COVID-19 treatment. We have also reviewed different therapeutic strategies with plant-based natural products that may be used to cure patients infected with SARS-CoV-2 and post-infection syndrome.
\end{abstract}

Amjad Husain

husainamjad@gmail.com

1 Department of Chemistry and Biochemistry, The University of Arizona, Old Chemistry 226, Tucson, AZ 85721, USA

2 Amity Stem Cell Institute, Amity Medical School, Amity University Haryana, Amity Education Valley Panchgaon, Manesar (Gurugram), Haryana, India

3 Centre for Science \& Society, Indian Institute of Science Education and Research (IISER), Bhopal, Madhya Pradesh, India

4 Innovation and Incubation Centre for Entrepreneurship, Indian Institute of Science Education and Research (IISER), Bhopal, Madhya Pradesh, India 


\section{Graphical abstract}

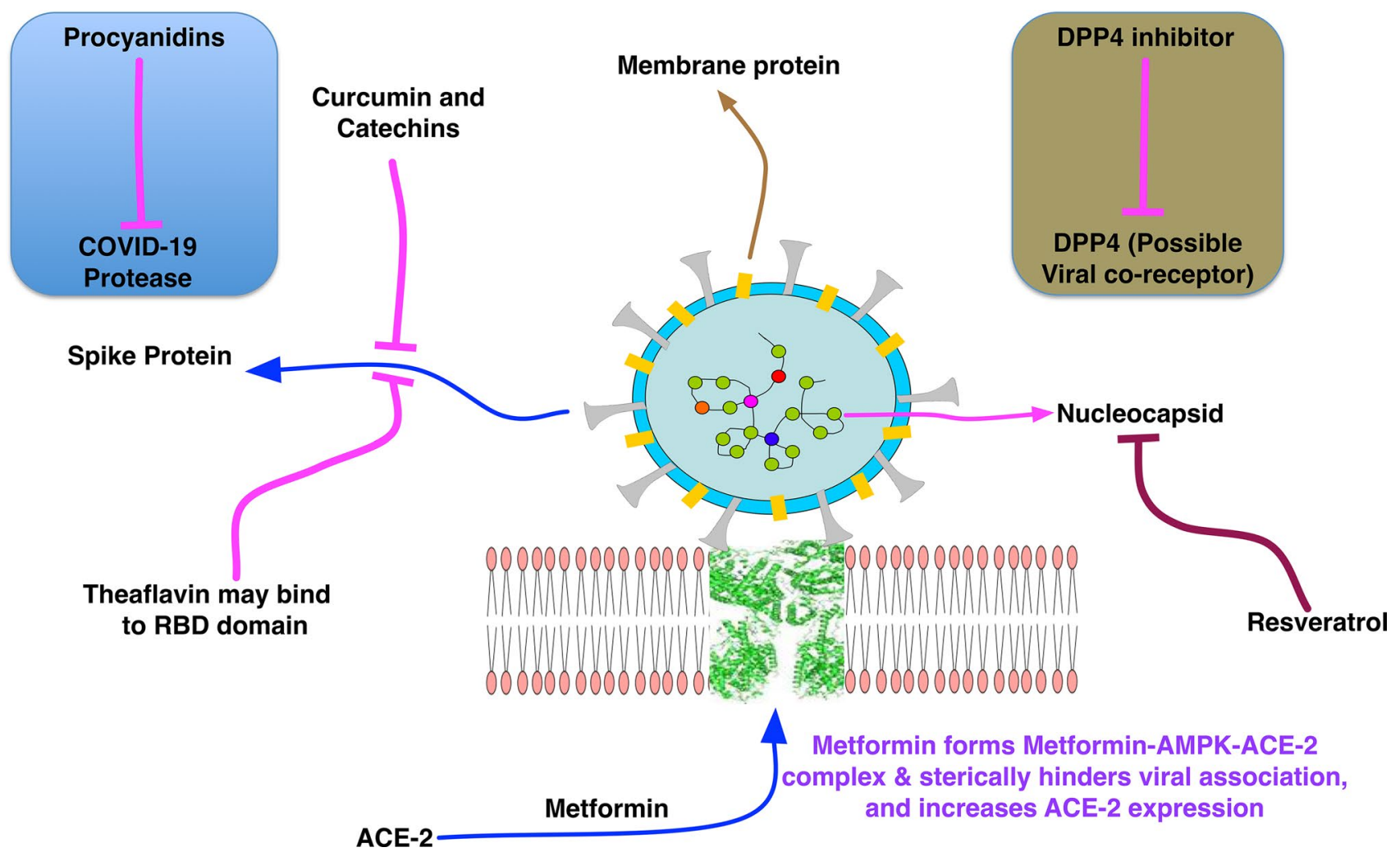

Keywords Human SARS coronavirus-2 $\cdot$ Diabetes $\cdot$ Aging $\cdot$ Resveratrol $\cdot$ Curcumin $\cdot$ Catechins $\cdot$ Metformin $\cdot$ Polyphenols and ACE-2

\begin{tabular}{ll}
\multicolumn{2}{l}{ Abbreviations } \\
SARS-CoV-2 & SARS coronavirus-2 \\
COVID-19 & Coronavirus-induced disease-2019 \\
IL & Interleukins \\
IFN & Interferon \\
ACE-2 & Angiotensin-converting enzyme-2
\end{tabular}

\section{Introduction}

Coronaviruses represent a group of viruses having singlestranded positive-sense RNA genome which causes disease in mammals and birds. The current COVID-19 pandemic is caused by human SARS coronavirus-2 (SARS-CoV-2), which is already known globally for the severe infection and high morbidity and mortality rates due to the lack of effective therapeutic drugs. The causative agent of this pandemic, SARS-CoV-2 consists of four proteins (Fig. 1) namely nucleocapsid protein, spike protein, envelope protein, and membrane protein [1]. Nucleocapsid proteins (Fig. 1A) are associated with the RNA genome. Spike proteins (Fig. 1B) have a multifunctional role in the coronavirus infection cycle and also facilitates virus entry to the host cells. Membrane proteins play a structural role in determining the shape of the viral envelope and participate in virus assembly. Envelope protein interacts with the membrane protein to form a virus envelope. The infection is initiated when the viral spike protein recognizes and binds to the host-cell receptor, Angiotensin-converting enzyme-2 (ACE-2), a key protein required for the viral infection [2]. The virus exclusively targets ACE-2-expressing cells and has a very high binding affinity as compared to other SARS Coronaviruses [3]. This binding is followed by cleavage of the spike protein by certain proteases (TMPRSS2 and furin) leading to the membrane fusion to the host cell. It facilitates viral genome entry into the host cell $[4,5]$. The virus takes over the host machinery to replicate its genome and facilitates the formation of a multi-subunit replicase-transcriptase complex (RTC), which transcribes to generate new viral RNA. Nested groups of sub-genomic RNAs (sgRNA) are generated by means of fragmented transcription, which translates to form the viral structural and accessory proteins. The proteins 
Fig. 1 A Structural overview of Coronavirus. B The X-ray crystal structure of spike protein (PDB id: 6ZDH). The spike protein plays a key role in virus-host interaction at the beginning of infection

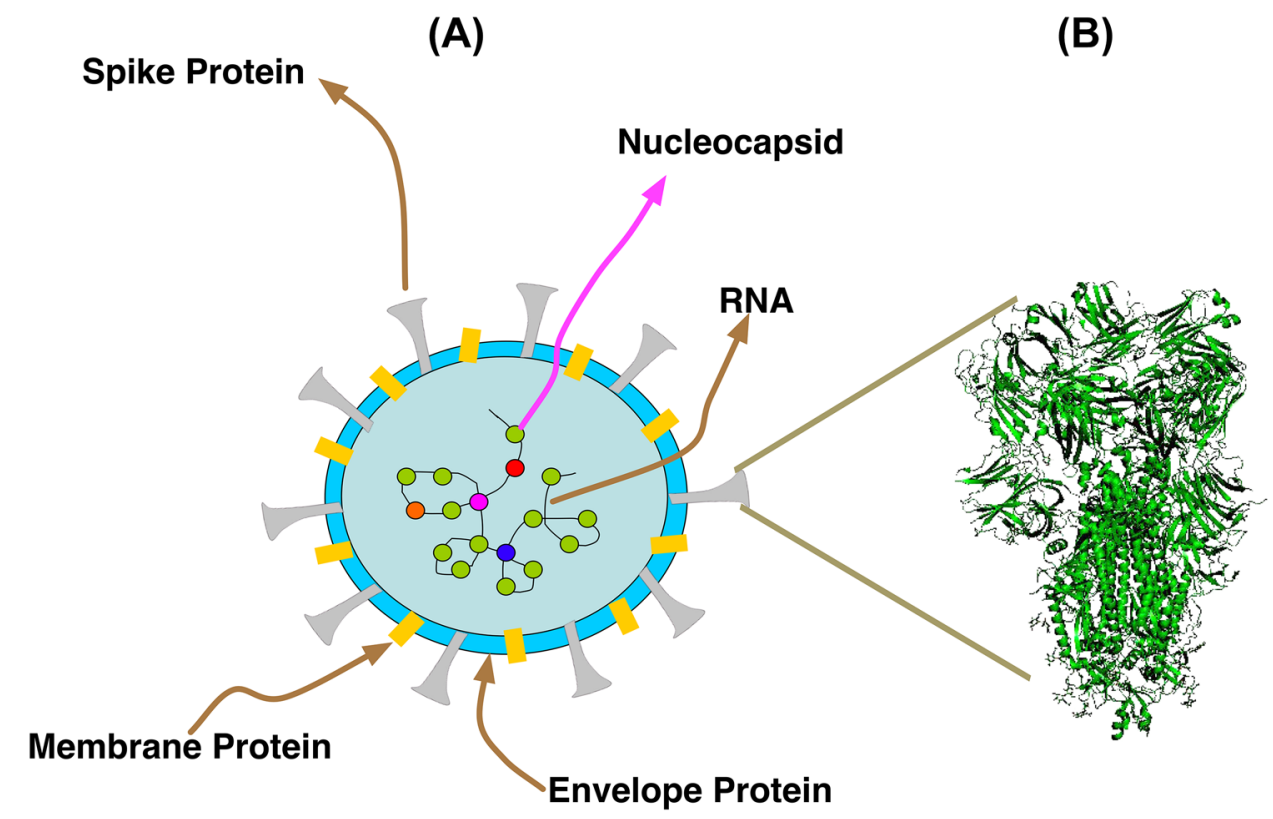

synthesis occurs at the ribosomes attached to the endoplasmic reticulum. The viral structural proteins viz. spike protein, membrane protein, and envelope protein are inserted into the endoplasmic reticulum. The nucleocapsid protein combines with the viral genome to form a nucleoprotein complex. Another complex called endoplasmic reticulum-Golgi intermediate compartment (ERGIC) facilitates new virion particles release [2]. The mature virion is generated in the Golgi apparatus, which is moved to the extracellular space through exocytosis [2, 6].

The COVID-19 pandemic is caused due to the infection with the newly recognized Human SARS-CoV-2 strain. The disease was first reported in Wuhan, China, in December 2019, and now, has been extended globally to more than 195 countries affecting more than 170 million people and has caused $>3.5$ million deaths. The causative virus can get transmitted from person to person via respiratory droplets or aerosol. Like most other coronaviruses, it spreads through the respiratory tract. The symptoms can vary from being asymptomatic to high fever, dry cough, sore throat, breathlessness, fatigue, and loss of taste or smell. The common methods used for the diagnosis of SARS-CoV-2 infection include RT-PCR of the oropharyngeal or nasopharyngeal sample or antibody test. Although the antibody test may also provide some information about the previous infection [7, 8], it fails to detect the infection at a very early stage. The RNA-based test RT-PCR is considered a gold standard of COVID-19 diagnosis as it picks up the early stage infections too. The RT-PCR offers highly standardized detection of the SARS-CoV-2 RNA; still, it is vulnerable to falsenegative diagnosis due to the absence of infected cells in the sample or inaccurate RNA extraction. Hence, alternative protocols were also recently proposed to minimize falsenegative detection [8].

Antiviral agents that would be developed in the future for the SARS-CoV-2 may target specific viral components, but resistance to these antiviral drugs is likely to appear due to multiple mutations in the viral RNA and new viral variants. Therefore, therapeutics targeting the host-cell component that regulates viral replication, virion assembly, and its release may add value for future drug development.

\section{Immune response during the COVID-19}

The understanding of the immune response to the coronavirus is very limited as of now; however, the outcome is expected to be similar to other coronavirus infections based on the sequence homology of Human SARS-CoV-2 to other coronaviruses and conservation of immune signaling. Pattern recognition receptors (PRRs) identify pathogenassociated molecular patterns (PAMP) in response to the viral infection. Toll-like receptors, (TLR) and NOD-like receptors (NLR) are some of the examples of PRRs, which get activated in response to the viral infection. The triggering of these receptors is followed by the production of the cytokines. Interferon types I and III are the most significant cytokines that can limit the COVID-19 infection. Other cytokines include pro-inflammatory tumor necrosis factoralpha (TNF- $\alpha$ ), and interleukin-1 (IL-1), IL-6, and IL-18 [9, 10]. A 'cytokine storm' due to the pro-inflammatory response has been also observed in coronavirus-infected patients, and the pathogenicity of the disease is correlated to the imbalance of interferon response. More data and studies are required to understand the correlation of host antiviral and inflammatory response. 
There are various co-morbidities associated with COVID19 infection that include diabetes, cardiovascular disease, and hypertension [10, 11]. Several reports and clinical studies suggest that the COVID-19 patients with diabetes need more intensive care and have lesser chances of recovery [12-14]. Another factor that has been associated with a higher viral infection and the severity of the disease is aging. Elderly people have a weak immune system, and they are prone to the severity of viral infection in general, and in the case of COVID-19 infection, the severity and mortality are high in such patients. Interestingly, there are studies that have found accelerated aging in diabetic patients. Since drug development is a time-taking process that requires years going through the different stages of pre-clinical and clinical development, the existing natural compounds can be explored for the treatment based on scientific rationale. Therefore, we also reviewed plant-based natural compounds that have been shown to have potential antiviral, anti-diabetic, and anti-aging effects. Most importantly, these compounds are safe to consume with no life-threatening byproducts or chemical reactions in general.

\section{Diabetes mellitus, aging, and COVID-19}

Notably, old age patients with diabetes are at a very high risk to develop severity of the infection with SARS-CoV-2. It has also been reported that COVID-19 can switch on the diabetes in individuals with no previous history of diabetes [8]. There is a bidirectional association between diabetes and COVID-19, as the COVID-19 not only severely affects the hyperglycemia pathophysiology in diabetic patients but also makes diabetic patients 50\% more prone to develop the risk of serious complications and death as compared with non-diabetic patients. In addition, the likelihood of developing diabetes increases many fold in the post-COVID-19 syndrome (where the symptoms of the disease persist for a long time even after the patient is tested negative for the virus) [15].

Patients with diabetes are at a high risk of other infections and disease including cardiovascular disease, delayed wound healing, foot infections, and eye-related disorders $[16,17]$. In diabetic individuals, the immune system gets weakened, and the disorganized release of cytokines happens by T-cells and macrophages that result in multi-system dysregulation. Synthesis and secretion of Interferon (IFNalpha and IFN-gamma) from CD8 + T-cells are reduced, and there is a decrease in the population of new dendritic cells and natural killer (NK) cells that further impairs the immune response. In addition, the virus may survive in higher glucose levels making it harder to eliminate the disease [18]. Also, a lower level of IFN-I (an important factor for antiviral immune response) in diabetic patients is likely to be the reason for the higher mortality of COVID-19 patients with pre-existing diabetes. Interferons can potentially be used for the treatment of viral infections and encouraging results have been observed when IFN-alpha nasal drops were used to prevent the infection $[10,19]$. It would be interesting to see if interferon treatment of COVID patients results in different response in diabetic vs non diabetic group.

Diabetes also expedites the biological aging process and the frequency of diabetes also surges with age with about 30 percent of elderly people suffering from diabetes [20]. There are different mechanisms proposed that explain how diabetes kicks in elderly people and relates to aging. The process of non-enzymatic glycation plays a significant role in the progress of aging. The production of advanced glycation end products (AGEs), which are formed due to glucose and protein chemical interaction, accelerates diabetes $[21,22]$. They accumulate in the body and form crosslinks with other molecules in the cells. The other factors such as an increase in oxidative damage, the elevation of $\mathrm{Na} / \mathrm{K}$ ATPase activity, growth in capillary basement membrane thickening, and decrease in DNA unwinding rate in diabetics further catalyze biological aging. In addition, the association of diabetes with cardiovascular disease and hypertension adds up to the accelerated aging process.

There are multiple reports mentioned about increased oxidative stress in COVID-19 patients [23-25]. After virus enters the host cell, the immune system generates macrophage and dendritic cells, which generate ROS (reactive oxygen species). In individuals with diminished redox balance (pro-oxidant to anti-oxidant ratio), the ROS leads to the destruction of erythrocytes and neutrophil activation, which in turn results in respiratory bursts forming superoxide radicals and hydrogen peroxide. These superoxides and peroxides cause oxidative stress, which initiates "cytokine storm" and hence COVID-19 severity [23]. Oxidative stress also plays a crucial role in the pathogenesis and advancement of diabetes. Insulin resistance in cells occurs due to free radical formation by non-enzymatic glycation of proteins, glucose oxidation, and surge in lipid peroxidation [22]. The ROS generated due to oxidative stress downregulates insulin signaling and leads to insulin resistance. This ROS can lead to damage in cellular machinery by causing destruction to DNA, lipids, proteins, and other cellular components. The stacking up of these damaged components results in faster aging [26].

Aging can make it difficult to manage diabetes. The glucose tolerance drastically decreases with age in addition to the decline in the number of naive T-cells, and the ability to recognize the new antigen. Cytokines like IL-2 expression and signal transduction are also affected. Interferon-1 (IFN-1) expression is also reduced in aged individuals. The reduction in cell-mediated immune response further increases complications in old age people. It is well documented by now that elderly patients with COVID-19 are at 
a high risk of infection severity and fatality, with accelerated aging symptoms. It needs to be further evaluated if the biomarkers for biological aging and diabetes can be used to evaluate the intensity of the COVID-19.

Aging can also lead to several other diseases like Alzheimer's disease, which has a reciprocal pathophysiological relationship with diabetes mellitus [27]. Another intersecting aspect relating diabetes, aging and COVID-19 lies with gender statistics. Men are more susceptible to diabetes [28, 29] as well as aging [30], and a similar trend of high mortality of males is observed in COVID-19. Since there is a direct relationship between aging, diabetes, and COVID19 ; there is a possibility of using one therapy to control all three diseases at once. Here, we have discussed several therapeutic agents and natural compounds, which can be potentially explored for the treatment of these diseases.

\section{Potential therapeutic approaches for the treatment of COVID-19}

One of the most important aspects which have not been studied extensively is the effect of post SARS-CoV-2 infection in diabetic and elderly patients. Does the post-coronavirus syndrome accelerate biological aging in asymptomatic patients (about $70 \%$ of the infected people) and consequently decrease the lifespan? It is an important question to be addressed. There are reports, which showcase accelerated death of muscle cells and lead to cachexia during and even after the recovery from COVID-19 [31]. Cachexia, which is associated with multiple diseases including cancer [32], is also observed and is one of the hallmarks of aging and diabetes [32,33]. Since diabetes is known to enhance aging and susceptibility to viral infection, an effective drug that helps in the treatment against COVID-19 can be anti-diabetic and anti-aging which can serve as a prophylactic or a long-term solution to avoid the SARS-CoV-2 infection induced severity. Various therapeutic strategies using natural compounds with high safety profiles have been discussed below for possible treatment options for COVID-19.

\section{Polyphenols}

These are the group of chemical compounds which not only have large multiples of phenols as structural units, and abundant in natural resources like plants, but can also be synthesized chemically. Plant-based polyphenols (flavonoids, phenolic acid, and stilbenes) possess high anti-oxidant activity [34] and can help to lower blood sugar [35] levels. Several polyphenols like resveratrol, curcumin, catechins, and procyanidins have anti-diabetic properties. There are evidences that certain polyphenols have the potential to treat not only the viral infection [36], but also slow down the aging process [37-39]. More studies need to be done to

Natural Compounds as Anti-aging, Anti-diabetic, or Anti-viral Agents

A. Resveratrol
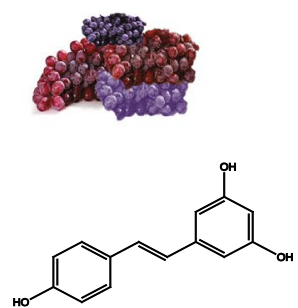

D. Procyanidin
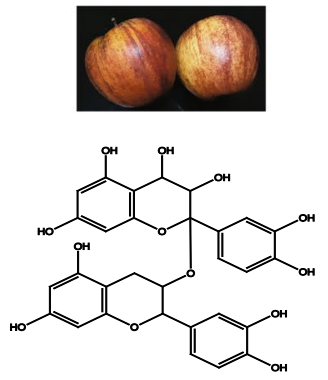

B. Curcumin
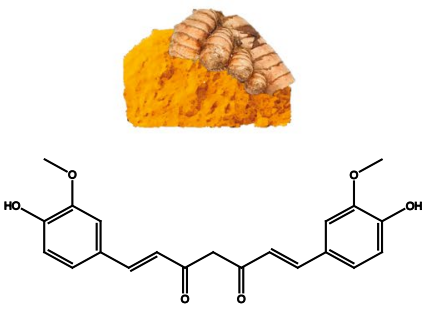

E. Theaflavin
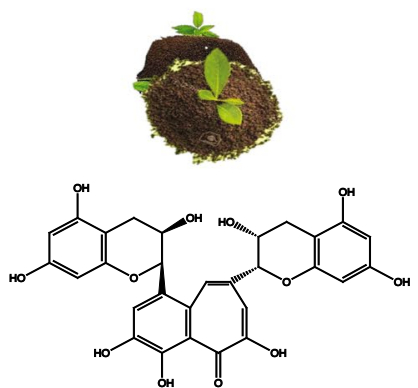

C. Catechin
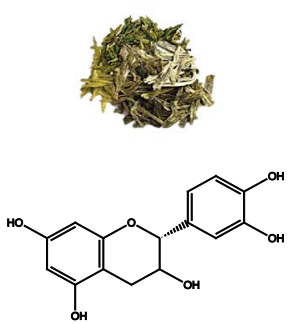

G. Dipeptidyl peptidase-4 Inhibitor

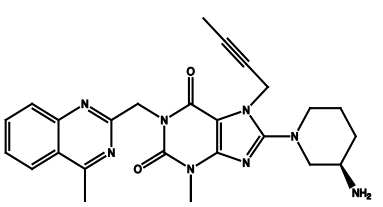

Fig. 2 A-G Chemical structure of plant-based natural compounds that have potential to treat COVID-19 along with diabetes and aging 
evaluate the potential of polyphenols to treat the COVID-19, but polyphenols may offer multiple potential agents that can be explored to prevent and treat the viral infection.

\section{Resveratrol}

Resveratrol (3,5,4'-trihydroxy-trans-stilbene) (Fig. 2A) is a polyphenol that can be obtained naturally from plants like blueberries, grapes, and cranberries, and also found in red wine. The in vitro studies have shown that resveratrol can potentially inhibit the replication of emerging respiratory viruses, especially the MERS virus [40, 41]. Resveratrol is also known to be effective against inflammation by impeding the TNF- $\alpha$-induced effect and IL-6 mRNA expression [42]. Furthermore, resveratrol has been shown to downregulate the TNF- $\alpha$ pre-mRNA splicing in fishes to reduce inflammation. In addition, it halts phosphodiesterases (PDEs) (mainly PDE3B, PDE8A, and PDE10A) activity, which breaks down cyclic adenosine monophosphate (cAMP) molecules. The cAMP is known to play a crucial role in glucose and insulin secretion. The increase in intracellular cAMP promotes cell signaling pathways which increase insulin and enhance $\beta$-cell functions. Resveratrol has been shown to have antiviral activity by targeting the nucleocapsid protein of the virus.

Resveratrol modulates sirtuins (SIRT1) activity, which controls aging-related pathways. Through a signaling cascade, it leads to an increase in mitochondrial activity and decreases the effect of oxidative stress in the body. It also inhibits cellular adhesion molecules and inflammatory markers such as NF-кB. It is also associated with an increase in $\mathrm{NO}$ (nitric oxide) production, which boosts vasorelaxation. It also improves fat mobilization, fatty acids oxidation, lipolysis, weight loss and overall anti-aging effects [43].

\section{Curcumin}

Curcumin (diferuloylmethane) (Fig. 2B) is a natural polyphenolic compound present in turmeric. It exhibits antioxidant activity, suppresses NF- $\mathrm{kB}$ activity, increases p53 activity, and therefore, anti-carcinogenic in nature [44, 45]. It has been shown that it enhances insulin resistance and ameliorates hyperglycemia. It upregulates adiponectin, which regulates glucose and fatty acid oxidation. Its antiinflammatory and anti-oxidant properties make it a potential anti-aging compound.

Curcumin has the potential to inhibit SARS-CoV-2 infections as shown in some of the in silico studies [46-48]. However, further studies are required to evaluate curcumin's potential as an antiviral drug. It is suggested that at the molecular level, it acts through two different mechanisms. In the first mechanism, it inhibits the viral spike protein and host protein interactions. It has binding affinities to both the viral S-protein (receptor-binding domain) and the host protein ACE-2, a protein required for the binding of virus with the host cells. Obstruction in the host-viral interaction can prevent the multiplication and spread of the virus in the body. Curcumin can further modulate diabetes by increasing the ACE- 2 protein expression, which promotes increased secretion of insulin in the pancreas.

Curcumin also has the potential to regulate the proinflammatory effects of the Angiotensin II-AT1 receptorsignaling pathway, which decrease respiratory distress and, therefore, can be used to cure the viral infection. It further modulates the activity of pro and anti-inflammatory cytokines such as IL-6, IL-8, and IL-10, and mitigates the cytokine storm that happens during COVID-19 [49]. One of the functions of the cytokine includes the formation of the reactive oxygen species (ROS) by upregulating NADPH oxidase in leukocyte. Cytokine modulation and diabetes are also associated with each other as unusual production of cytokines like IL- 6 are related to insulin resistance [50]. Therefore, we have presented how curcumin can act against virus infection, diabetes, and aging through overlapping signaling pathway and common intermediaries.

\section{Catechins (flavanol)}

They are anti-oxidants like flavonoids that are present in tea, cocoa, and berries. Chemically these have two benzene rings, heterocyclic dihydropyran, and a ring with the hydroxyl group on carbon-3 moiety (Fig. 2C). Different types of catechins are present in tea but epigallocatechin3 -gallate (EGCG) found in green tea is one of the most promising catechins under consideration to treat COVID19 disease [51]. It has been previously proved successful against porcine reproductive and respiratory syndrome virus (PRRSV) infection [52]. Studies have shown that catechins can help in glucose homeostasis. Furthermore, they help in the translocation of insulin-dependent GLUT4 transporters. Molecular simulation studies have shown that catechins have a dual-binding affinity to viral S-protein and ACE-2 receptors. In addition, the tea catechins have shown to help in reducing oxidative stress in erythrocytes by mitigating malondialdehyde (MDA) levels and protecting the membrane -SH group from oxidation.

\section{Procyanidins}

Procyanidins are formed from oligomerization or polymerization of flavonoids (catechins, epigallocatechin, epicatechin, and gallocatechin) (Fig. 2D). They decrease the activity of IL- 6 and MCP-1 and upregulate adipokine and adiponectin concentration, which exhibits anti-inflammatory activity. They are also known to induce GLUT4 translocation 
by upregulating AMP kinase (AMPK) and insulin signaling pathways [53].

Procyanidins have been shown to have antiviral properties against SARS-CoV-2 infection. Computational studies have shown that procyanidin B2 has an affinity for SARS$\mathrm{CoV}-2$ protease [54]. Since the proteases are involved in the proteolytic processing of polyprotein and play a significant role in the replication of the virus within the host cells, the binding affinity with procyanidins may help in controlling the SARS-CoV-2 infection. Procyanidins along with resveratrol exhibit anti-aging properties by regulating the activity of AMPK and sirtuin-1, which are involved in pathways related to metabolism modulation.

\section{Theaflavin}

Theaflavins are polyphenols (Fig. 2E) that demonstrate high anti-oxidant and anti-hyperglycemic activity. It boosts hexokinase, pyruvate kinase, and glucose-6-phosphate dehydrogenase enzyme activities involved in glucose metabolism. It upregulates glycogen synthase, which ameliorates glycogen content of liver and muscles [55]. It further downregulates gluconeogenesis and glycogenolytic enzyme activities.

These polyphenols have the potential to bind with the viral spike protein (receptor RBD protein) and inhibit hostviral association as shown by computational studies [56], but in vitro and clinical studies need to be conducted to evaluate the efficacy of these compounds against the virus. The anti-oxidant and free radical scavenging properties of these compounds [57] qualify them to become potential antiaging compounds. They modulate erythrocyte malondialdehyde (MDA), intracellular-reduced glutathione (GSH), and plasma membrane redox system (PMRS) enzymes.

\section{Metformin}

Metformin is an anti-hyperglycemic drug that shows pleiotropic functions in human with no major side effects (Fig. 2F). Metformin is primarily used for the treatment of diabetes and has been tested for the treatment of liver disease, cardiovascular disease, obesity, and cancer [58]. The drug activates AMPK in liver cells resulting in the uptake of glucose in muscles. Metformin also inhibits TNF- $\alpha$ which leads to the anti-inflammatory response and hence control, the "cytokine storm" that is one of the key events during the COVID-19 severity. There are several different ways by which metformin can inhibit coronavirus activity [59].

Metformin may inhibit virus-host-cell interaction by forming the Metformin-AMPK-ACE-2 complex. The binding of virus spike protein with ACE-2 is the first step of infection. The receptor-binding domain (RBD) of spike protein and ACE-2 interact with each other during the normal viral infection. Metformin inhibits this viral-host-cell association as well as increases the ACE-2 protein activation. Metformin phosphorylates the ACE-2 protein by activating AMPK. This phosphorylation sterically hinders the viral protein binding with the ACE-2 receptor and, thus, interrupts their association $[59,60]$.

In addition, metformin exhibits anti-aging activity, which is also attributed to increased expression of ACE-2 protein [59]. The ACE- 2 protein catalyzes the conversion of Angiotensin II to Angiotensin, which is known to decrease ROS generation and apoptosis. Therefore overall, metformin is a potential candidate that can be used for anti-diabetic, antiviral, and anti-aging effects with a good safety profile [60].

Metformin may also inhibits the mTOR pathway which is a key pathway involved in the pathogenesis of several other viruses [61]. Metformin inhibits this pathway by modulating the liver kinase B1 (LKB1) that negatively controls the mTOR signaling cascade [62]. Metformin also inhibits AKT (Protein Kinase B) expression, which is an activator of the mTOR pathway. Metformin exhibits anti-aging potential and further downregulates frailty, and increases longevity in elderly people.

\section{Rapamycin}

Since the mTOR pathway is associated with several viral infections, the mTOR inhibitor rapamycin has been recently proposed for the treatment of COVID-19 disease [63]. Rapamycin can be effective in the treatment of COVID-19 through its anti-aging and anti-obesity effects along with its inhibitory effect on the synthesis of proteins and lipids [63]. Although rapamycin was originally described as an anti-fungal agent, it was later discovered that it also acts as an immunosuppressive agent. It has been in use for the treatment of renal cancer and other solid tumors. In various studies, it has been shown that rapamycin suppresses the viability and proliferation of tumor and endothelial cells. Over the last decade, it has become clear that reducing the pharmacological inhibition of mTORC1 by rapamycin delays aging. Rapamycin has shown to be effective against mouse models of age-related diseases, including Alzheimer's disease. Rapamycin-mediated increase in life span of mice has been observed at doses without the noticeable side effects. Being an immunosuppressant, it also decreases the secretion of cytokines such as IL-6, IL-2, and IL-10 and can potentially reduce the intensity of cytokine storm in COVID-19 patients. Also, there are multiple in silico studies that identify mTOR as one of the central molecules in COVID-19 based on data from other human coronavirus infections using the network-based drug repurposing model. Therefore, using a low dosage of rapamycin as a therapy to treat COVID-19, may be considered, however, first, it has to 
be tested on viral infected cells, in pre-clinical studies and followed by the clinical trials on COVID-19 patients.

\section{Dipeptidyl peptidase-4 (DPP4) inhibitors}

Dipeptidyl peptidase-4 (CD26 or adenosine deaminase complexing protein 2) is a type II transmembrane glycoprotein that is expressed usually on cell surfaces and known to be involved in immune regulation, signal transduction, and apoptosis. It has a strong binding affinity to the virus and may act as a co-receptor along with ACE-2 protein to initiate the infection. It is well studied that the beta-propeller domain of DPP4 binds to the spike protein of the MERS-CoV virus, which has significant similarity to other coronaviruses. The DPP4 downregulates GLP-I (gut-derived incretins) and GIP (glucose-dependent insulinotropic polypeptide), which modulates insulin secretion. Thus, inhibition of DPP4 leads to an increase in insulin secretion. It further triggers T-cell activation, which results in an increase in CD86 expression, NF-kB pathway, cell adhesion, chemotaxis modulation, and apoptosis. A DPP4 inhibitor, linagliptin (Fig. 2G), has been studied for its anti-aging properties. Although initial studies underscored its value to be less promising against COVID19 treatment, more data and analysis need to be done to come to a conclusion.

\section{Cyanobacterial and phytochemical products}

Certain metabolites (lectins, sulfated polysaccharides, alkaloids) found in cyanobacteria are known to have antiviral activities and can be used as promising therapeutics agents against coronavirus infection. In addition, there are phytochemical products such as spermidine and spermine, which can target mTOR and autophagosomal signaling pathways and can be explored for the treatment of coronavirus infection [64]. Some of the phytocompounds such as quercetin derivatives and flavonoids target the virus proteases, which are required for viral spread [65]. They can also act as a potential anti-diabetic agent. Taken all together, these natural products must be explored as an alternative to the synthetic drugs for COVID-19 treatment, which can also cure diabetes and aging.

\section{Targeting virus-host membrane interactions with natural compounds}

Membrane lipids composition [66, 67] or micro-domains like lipid rafts play an important role in regulating diabetes, aging, and coronavirus infection $[68,69]$. Notably, the human ACE-2 protein is a transmembrane protein with residues $741-761$ forming a transmembrane domain. The role of the transmembrane domain has not been explored in detail in the viral infection cycle [70]. Looking back at the studies published on SARS Coronavirus and related viruses, there are evidences that showed interaction of viral proteins with transmembrane regions of variety of host proteins. The Orf3a protein of SARS coronavirus is known to interact with caveolin [71]. In case of HIV-1 infection, the transmembrane domain of $\mathrm{VpU}$ protein of HIV-1 is known to form an oligomer, and the oligomerization appears to be either the Golgi region or intracellular vesicles [72]. There are studies that showed loss of cell surface molecules CD80 and CD86 in antigen-presenting cells during HIV infection and suggest an involvement of the membrane [73]. In addition, the HIV1-Vpu protein interacts with CD74 and modulates major histocompatibility complex class II presentation that requires the involvement of the membrane and ER-Golgi complex [74].
Fig. 3 Molecular-docking studies of ACE-2 binding with (A) wild-type spike protein and (B) mutated spike (D614G)ACE-2 protein. The inset shows (A) the D614 and (B) D614G residues. The difference in free energy change explains why the mutated form of SARS-CoV-2 is more contagious

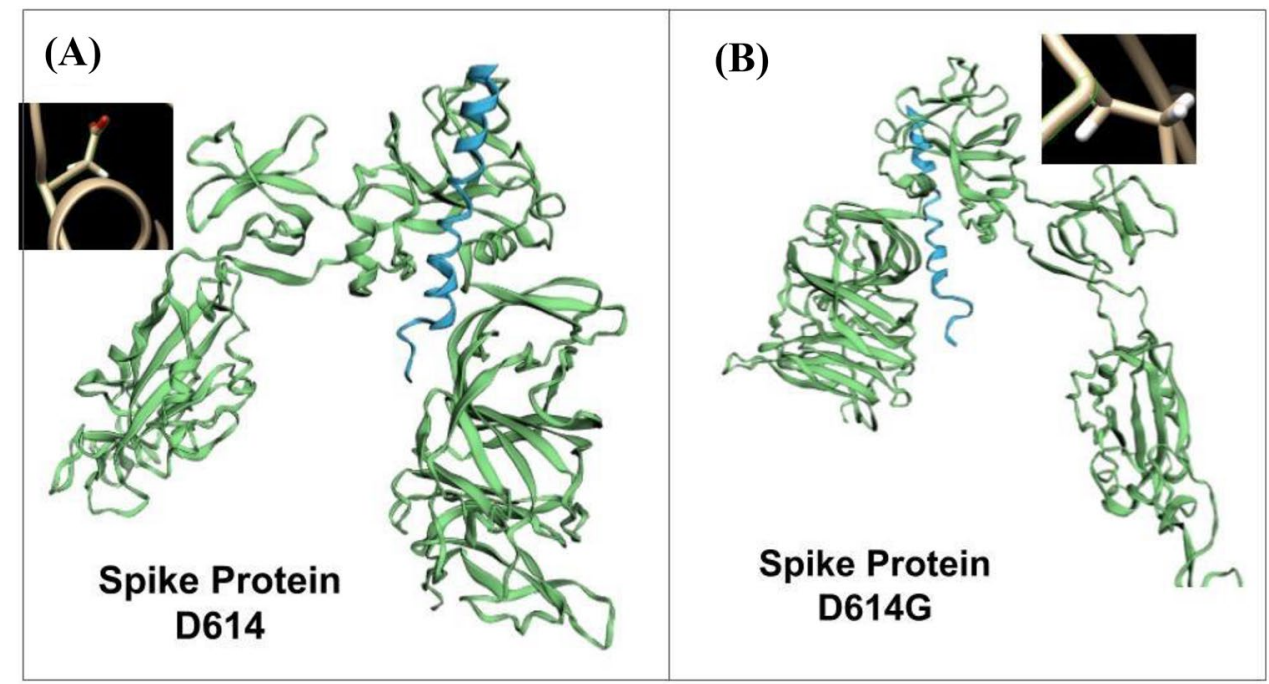




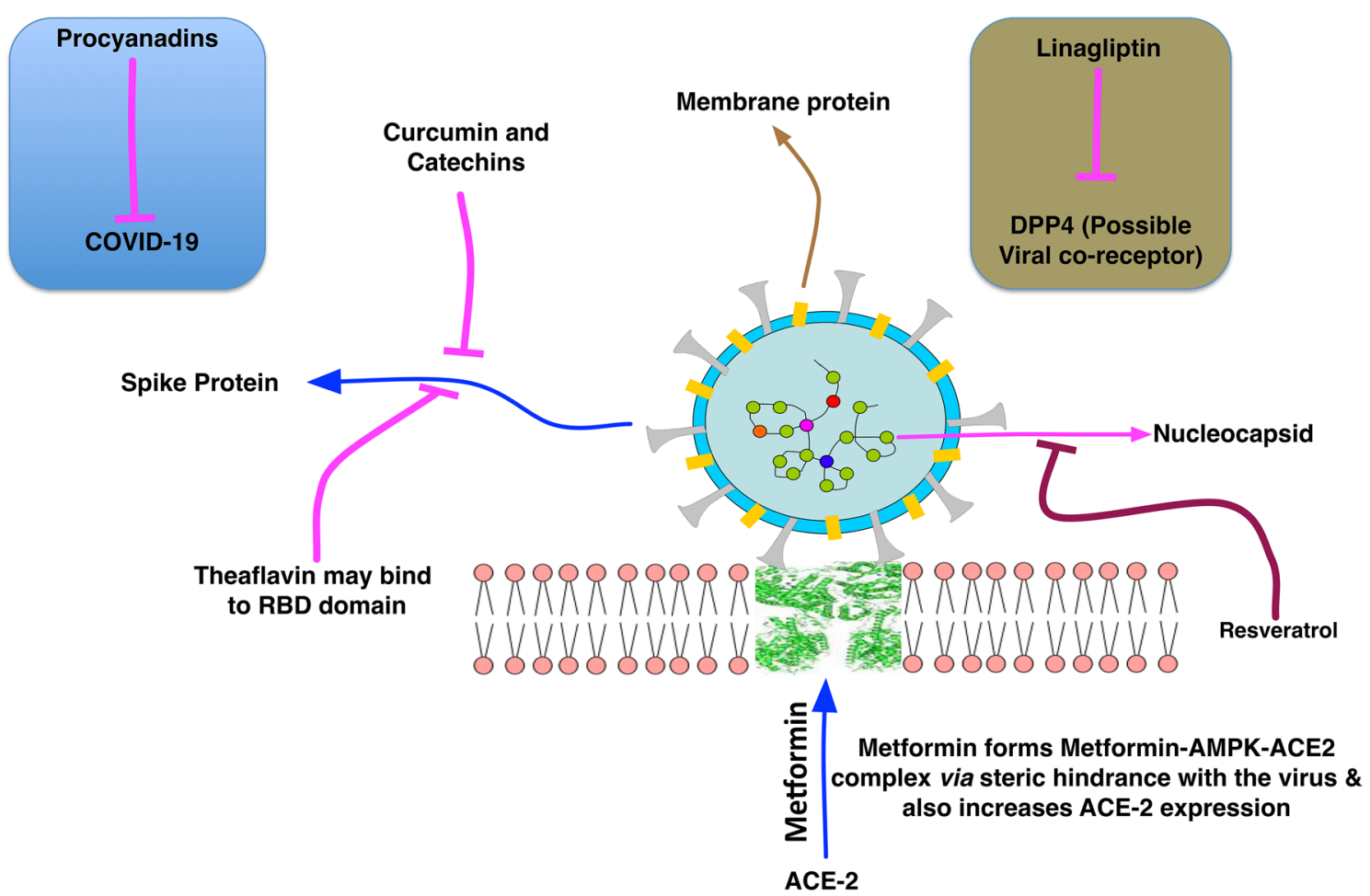

Fig. 4 Overview of existing drugs and natural compounds and their mechanism of action towards the treatment of COVID-19. These compounds also have the potential to show anti-diabetic and anti-aging effects with high safety profiles

Using in silico methods, we explored the possible role of the ACE-2 transmembrane domain in COVID-19 viral infection. We hypothesized that the mutation in SARSCoV-2 (D614G) might affect the binding to the ACE-2 transmembrane domain and mutant form may become more contagious. To test our hypothesis, we obtained a predicted model for ACE-2 (transmembrane domain) and viral spike protein using swissdock and I-TASSER servers, respectively. Further, protein-protein interactions modeling and binding-free energy of the complex (for interactions of ACE-2 with native and D614G mutated spike protein) were obtained using the HawkDock server. The binding-free energy change for human ACE-2 and viral spike protein association for wild-type and D614G mutant was determined to be $-40.6 \mathrm{kcal} / \mathrm{mol}$ and $-48.0 \mathrm{kcal} /$ mol, respectively (Fig. 3). The big difference in binding affinity for the wild type vs mutant form explains why the latter may become more contagious. Our computational study also demonstrates that membrane lipids can play an important role in coronavirus infectivity. Natural compounds such as flavonoids can affect the regulation of lipid raft formation and viral replication. Thus, these compounds can be used to possibly target membrane lipids and hence for controlling the virus spread.

\section{Conclusion}

COVID-19 disease, a pandemic, caused by human SARS CoV-2, has affected individuals in more than 196 countries with more than 170 million cases and more than 3.5 million deaths. The outbreak of COVID-19 has exposed the world population to viral infections, and the diabetics and elderly people are facing the severity with high mortality rates as compared to younger population. In addition, how the individual's health got affected after the recovery in the long term is unknown. There is growing evidence that asymptomatic patients and healthy individuals who have been exposed to the virus once may have long-term effects [15]. How does the infection affect accelerated aging or onset of diabetes, needs to be further studied.

At the molecular level, there are intersecting pathways regulating diabetes, aging, and COVID-19. Oxidative stress and lowering of the immune response are often associated with all three diseases. The complications of the diseases lead to the onset of numerous other diseases; for example, diabetes is also associated with cardiovascular problems, eye diseases, neuropathy, and nephropathy, along with other long-term complications [17]. Similarly, aging increases the chances of cardiovascular disorders, cancer, and arthritis. The coronavirus infection may also lead to septic shock, 
acute respiratory distress syndrome (ARDS), mental distress, and joint pain. Hence, it is crucial to study the intersection of these diseases and find ways to manage and cure these diseases at the appropriate time. An ideal therapeutic candidate should be able to target the common pathways and control these diseases together while inhibiting the replication of the SARS-CoV-2, its assembly, and release from the infected cells. Also, different key pathways including AKT and The mTOR pathway are known to regulate diabetes and aging and have been also shown to get modulated by viruses, such as Influenza A, for the virus release from the cell. Many viruses including human herpesvirus (HHV) require the activation of $\mathrm{AKT}$, a pro-survival protein kinase, for the propagation of virus-infected cells. The multiplication of virus-infected cell also requires activation of mTOR pathway. In several studies, the mTOR inhibitor Rapamycin has also been shown to inhibit viral protein synthesis, and may inhibit an essential step required for the synthesis of the new virus particle.

In addition, there are naturally existing compounds that affect the binding of the virus to host receptors and affect molecular interactions required for virus replication and release. These natural compounds bind well with ACE-2 and other required proteins essential for the infection. We have also suggested that the catechin derivatives may act as potential inhibitors to SARS-CoV-2 infection and help controlling the COVID-19 severity. Overall, this needs to be understood and considered to shortlist the effective therapeutics from the existing pool of potential compounds. Various plant-based natural compounds such as resveratrol, curcumin, catechin, procyanidins, theaflavin, and existing drugs like metformin, and inhibitors of DPP4 (Fig. 4) are the potential candidates that can be tested for the treatment of COVID-19 as shown by various in silico, in vitro, and in vivo studies. With these existing molecules, there is a need to conduct extensive experiments to test their potential in the treatment of COVID-19 and post-coronavirus syndrome.

Acknowledgements We acknowledge the support and funding provided by the Indian Institute of Science Education and Research for this publication.

Funding MKK is the recipient of the TARE fellowship (Grant \# TAR/2018/001054) from the Science and Engineering Research Board (SERB), Department of Science and Technology, Government of India.

Data availability All data generated and analyzed during our study are included either in the published article or supplementary data associated with the manuscript.

\section{Declarations}

Conflict of interest There are no competing interests or conflict of interest in regards to the data presented in the manuscript. Dr. Udeep
Chawla and Dr. Amjad Husain (corresponding author) have contributed equally for the manuscript.

Ethical approval and consent to participate Not applicable because the data included in the study were collected form published studied on COVID-19. The study does not involve any human subjects, tissue samples, or cell lines.

Informed consent All the authors agreed and provided their consent for the publication of this review article.

Consent for publication All authors' consent to the publication of the manuscript. All figures or tables are original, so there was no requirement of taking permission or consent from anyone.

\section{References}

1. Seah I, Su X, Lingam G (2020) Revisiting the dangers of the coronavirus in the ophthalmology practice. Eye (Lond) 34:1155-1157

2. Jafary F, Jafari S, Ganjalikhany MR (2021) In silico investigation of critical binding pattern in SARS-CoV-2 spike protein with angiotensin-converting enzyme 2. Sci Rep 11(1):6927. https://doi. org/10.1038/s41598-021-86380-2

3. Ciaglia E, Vecchione C, Puca AA (2020) COVID-19 Infection and Circulating ACE2 Levels: Protective Role in Women and Children. Front Pediatr 8:206

4. Hoffmann M et al (2020) SARS-CoV-2 Cell Entry Depends on ACE2 and TMPRSS2 and Is Blocked by a Clinically Proven Protease Inhibitor. Cell 181:271-280.e278

5. Sahu AK, Sreepadmanabh M, Rai M, Chande A SARS-CoV-2: phylogenetic origins, pathogenesis, modes of transmission, and the potential role of nanotechnology. Virusdisease. https://doi. org/10.1007/s13337-021-00653-y. Epub ahead of print. PMID: 33644261; PMCID: PMC7897733 (2021).

6. Sreepadmanabh M, Sahu AK, Chande A (2020) COVID19: Advances in diagnostic tools, treatment strategies, and vaccine development. J Biosci. https://doi.org/10.1007/ s12038-020-00114-6

7. Alanagreh LA, Alzoughool F, Atoum M (2020) The human coronavirus disease COVID-19: its origin, characteristics, and insights into potential drugs and its mechanisms. Pathogens 9(5):331

8. Husain A, A novel approach to minimize the false negative COVID-19 diagnosis by inclusion of specific cell markers and multiple sample collection. MethodsX 8, 101270 (2021).

9. Li G et al (2020) Coronavirus infections and immune responses. J Med Virol 92:424-432

10. Mazucanti CH, Egan JM (2020) SARS-CoV-2 disease severity and diabetes: why the connection and what is to be done? Immun Ageing 17:21

11. Huang $\mathrm{C}$ et al (2020) Clinical features of patients infected with 2019 novel coronavirus in Wuhan. China Lancet 395:497-506

12. Apicella $\mathrm{M}$ et al (2020) COVID-19 in people with diabetes: understanding the reasons for worse outcomes. Lancet Diabetes Endocrinol 8:782-792

13. Riddle MC et al (2020) COVID-19 in people with diabetes: urgently needed lessons from early reports. Diabetes Care 43:1378-1381

14. Abdi A, Jalilian M, Sarbarzeh PA, Vlaisavljevic Z (2020) Diabetes and COVID-19: A systematic review on the current evidences. Diabetes Res Clin Pract 166:108347

15. Rubino F et al (2020) New-onset diabetes in Covid-19. N Engl J Med 383:789-790 
16. Ali I (2021) Syndemics at play: chronic kidney disease, diabetes and COVID-19 in Pakistan. Ann Med 53:581-586

17. Grundy SM, Benjamin IJ, Burke GL, Chait A, Eckel RH, Howard BV, Sowers JR (1999) Diabetes and cardiovascular disease: a statement for healthcare professionals from the American Heart Association. Circulation 100(10):1134-1146

18. Hoagland DA et al (2021) Leveraging the antiviral type I interferon system as a first line of defense against SARS-CoV-2 pathogenicity. Immunity 54:557-570.e555

19. Hasan A, Al-Ozairi E, Al-Baqsumi Z, Ahmad R, Al-Mulla F (2021) Cellular and humoral immune responses in Covid-19 and immunotherapeutic approaches. Immunotargets Ther 10:63-85

20. Morley JE (2008) Diabetes and aging: epidemiologic overview. Clin Geriatr Med 24:395-405

21. Bierhaus A, Hofmann MA, Ziegler R, Nawroth PP (1998) AGEs and their interaction with AGE-receptors in vascular disease and diabetes mellitus. I AGE concept Cardiovasc Res 37:586-600

22. Rojas A, Morales MA (2004) Advanced glycation and endothelial functions: a link towards vascular complications in diabetes. Life Sci 76:715-730

23. Cecchini R, Cecchini AL (2020) SARS-CoV-2 infection pathogenesis is related to oxidative stress as a response to aggression. Med Hypotheses 143:110102

24. Cekerevac I et al (2021) Predicting severity and intrahospital mortality in COVID-19: the place and role of oxidative stress. Oxid Med Cell Longev 2021:6615787

25. Laforge $\mathrm{M}$ et al (2020) Tissue damage from neutrophil-induced oxidative stress in COVID-19. Nat Rev Immunol 20:515-516

26. Styskal J, Van Remmen H, Richardson A, Salmon AB (2012) Oxidative stress and diabetes: what can we learn about insulin resistance from antioxidant mutant mouse models? Free Radic Biol Med 52:46-58

27. Ashraf GM et al (2021) Dissecting Sex-Related Cognition between Alzheimer's Disease and Diabetes: From Molecular Mechanisms to Potential Therapeutic Strategies. Oxid Med Cell Longev 2021:4572471

28. Espeland MA, Yassine H, Hayden KD, Hugenschmidt C, Bennett WL, Chao A, Neiberg R, Kahn SE, Luchsinger JA (2021) Action for Health in Diabetes (Look AHEAD) Research Group. Sex-related differences in cognitive trajectories in older individuals with type 2 diabetes and overweight or obesity. Alzheimers Dement (N Y). 7(1):e12160. https://doi.org/10.1002/trc2.12160. PMID: 33860069; PMCID: PMC8033410.

29. Ghachem F, Dufour T, Flp P, Gaudreau AA (2021) Cohen, Effects of Sex and Physical Activity Level on Serum Biomarker-Based Physiological Dysregulation: The Impact to Predict Frailty and Mortality in the Quebec NuAge Cohort. Gerontology 1-14

30. Blagosklonny MV (2010) Why men age faster but reproduce longer than women: mTOR and evolutionary perspectives. Aging (Albany NY) 2:265-273

31. Anker MS et al (2021) Weight loss, malnutrition, and cachexia in COVID-19: facts and numbers. J Cachexia Sarcopenia Muscle $12: 9-13$

32. Husain A, Hu N, Sadow PM, Nucera C (2016) Expression of angiogenic switch, cachexia and inflammation factors at the crossroad in undifferentiated thyroid carcinoma with BRAF(V600E). Cancer Lett 380:577-585

33. Ali S, Garcia JM (2014) Sarcopenia, cachexia and aging: diagnosis, mechanisms and therapeutic options - a mini-review. Gerontology 60:294-305

34. Jackson CE, Barohn RJ (1998) Diabetic neuropathic cachexia: report of a recurrent case. J Neurol Neurosurg Psychiatry 64:785-787

35. Xiao JB, Hgger P (2015) Dietary polyphenols and type 2 diabetes: current insights and future perspectives. Curr Med Chem 22:23-38
36. Vzquez-Calvo A, Jimnez de Oya N, Martn-Acebes MA, GarciaMoruno E, Saiz JC (2017) Antiviral properties of the natural polyphenols delphinidin and epigallocatechin gallate against the flaviviruses west nile virus, zika virus, and dengue virus. Front Microbiol 8:1314

37. Wu M et al (2020) Potential implications of polyphenols on aging considering oxidative stress, inflammation, autophagy, and gut microbiota. Crit Rev Food Sci Nutr 1-19

38. Chowdhury A, Nosoudi N, Karamched S, Parasaram V, Vyavahare N (2021) Polyphenol treatments increase elastin and collagen deposition by human dermal fibroblasts; Implications to improve skin health. J Dermatol Sci

39. Lo Muzio L, Bizzoca ME, Ravagnan G (2020) New intriguing possibility for prevention of coronavirus pneumonitis: Natural purified polyphenols. Oral Dis

40. Filardo S, Di Pietro M, Mastromarino P, Sessa R (2020) Therapeutic potential of resveratrol against emerging respiratory viral infections. Pharmacol Ther 214:107613

41. Lin SC et al (2017) Effective inhibition of MERS-CoV infection by resveratrol. BMC Infect Dis 17:144

42. A. M. Silva et al., Resveratrol as a natural anti-tumor necrosis factor- $\alpha$ molecule: implications to dendritic cells and their crosstalk with mesenchymal stromal cells. PLoS One 9, e91406 (2014).

43. Carrizzo A et al (2013) Resveratrol improves vascular function in patients with hypertension and dyslipidemia by modulating $\mathrm{NO}$ metabolism. Hypertension 62:359-366

44. Rahdar A et al (2021) Biochemical, ameliorative and cytotoxic effects of newly synthesized curcumin microemulsions: evidence from in vitro and in vivo studies. Nanomaterials

45. Kabir MT et al (2021) Potential role of curcumin and its nanoformulations to treat various types of cancers. Biomolecules

46. Babaei F, Nassiri-As1 M, Hosseinzadeh H (2020) Curcumin (a constituent of turmeric): new treatment option against COVID-19. Food Sci Nutr 8:5215-5227

47. Noor $\mathrm{H}$ et al (2021) Immunomodulatory and anti-cytokine therapeutic potential of curcumin and its derivatives for treating COVID-19 - a computational modeling. J Biomol Struct Dyn, $1-16$

48. Soni VK et al (2020) Curcumin, a traditional spice component, can hold the promise against COVID-19? Eur J Pharmacol $886: 173551$

49. Liu Z, Ying Y (2020) The inhibitory effect of curcumin on virus-induced cytokine storm and its potential use in the associated severe pneumonia. Front Cell Dev Biol 8:479

50. Senn JJ, Klover PJ, Nowak IA, Mooney RA (2002) Interleukin-6 induces cellular insulin resistance in hepatocytes. Diabetes 51:3391-3399

51. Calvo V, de JiménezOya N, Martín-Acebes MA, Garcia-Moruno E, Saiz JC (2017) Antiviral properties of the natural polyphenols delphinidin and epigallocatechin gallate against the flaviviruses west nile virus, zika virus, and dengue virus. Front Microbiol 8:1314

52. Zhao C, Liu S, Li C, Yang L, Zu Y (2014) In vitro evaluation of the antiviral activity of the synthetic epigallocatechin gallate analog-epigallocatechin gallate (EGCG) palmitate against porcine reproductive and respiratory syndrome virus. Viruses 6:938-950

53. Yamashita Y et al (2016) Procyanidin promotes translocation of glucose transporter 4 in muscle of mice through activation of insulin and AMPK signaling pathways. PLoS One 11:e0161704

54. Zhuang M et al (2009) Procyanidins and butanol extract of cinnamomi cortex inhibit SARS-CoV infection. Antiviral Res 82:73-81

55. Gothandam K, Ganesan VS, Ayyasamy T, Ramalingam S (2019) Antioxidant potential of theaflavin ameliorates the activities of 
key enzymes of glucose metabolism in high fat diet and streptozotocin - induced diabetic rats. Redox Rep 24:41-50

56. Mhatre S, Srivastava T, Naik S, Patravale V (2021) Antiviral activity of green tea and black tea polyphenols in prophylaxis and treatment of COVID-19: A review. Phytomedicine 85:153286

57. Fatima M, Kesharwani RK, Misra K, Rizvi SI (2013) Protective effect of theaflavin on erythrocytes subjected to in vitro oxidative stress. Biochem Res Int 2013:649759

58. Novelle MG, Ali A, Diéguez C, Bernier M, de Cabo R (2016) Metformin: a hopeful promise in aging research. Cold Spring Harb Perspect Med 6:a025932

59. Malhotra A, Hepokoski M, McCowen KC, Shyy JY-J (2020) ACE2, Metformin, and COVID-19. iScience 23:101425

60. Luo P et al (2020) Metformin treatment was associated with decreased mortality in COVID-19 patients with diabetes in a retrospective analysis. Am J Trop Med Hyg 103:69-72

61. Kuss-Duerkop SK et al (2017) Influenza virus differentially activates mTORC1 and mTORC2 signaling to maximize late stage replication. PLoS Pathog 13:e1006635

62. Shaw RJ et al (2004) The LKB1 tumor suppressor negatively regulates mTOR signaling. Cancer Cell 6:91-99

63. Husain A, Byrareddy SN (2020) Rapamycin as a potential repurpose drug candidate for the treatment of COVID-19. Chem Biol Interact 331:109282

64. Madeo F, Carmona-Gutierrez D, Kepp O, Kroemer G (2018) Spermidine delays aging in humans. Aging (Albany NY) 10:2209-2211

65. Bhattacharya R, Dev K, Sourirajan A (2021) Antiviral activity of bioactive phytocompounds against coronavirus: An update. J Virol Methods 290:114070

66. Chawla U et al (2016) A usual G-protein-coupled receptor in unusual membranes. Angew Chem Int Ed Engl 55:588-592
67. Chawla U et al (2021) Activation of the G-protein-coupled receptor rhodopsin by water. Angew Chem Int Ed Engl 60:2288-2295

68. Pilon M (2016) Revisiting the membrane-centric view of diabetes. Lipids Health Dis 15:167

69. Fecchi $\mathrm{K}$ et al (2020) Coronavirus interplay with lipid rafts and autophagy unveils promising therapeutic targets. Front Microbiol 11:1821

70. Kumar P et al (2021) Role of ACE2 receptor and the landscape of treatment options from convalescent plasma therapy to the drug repurposing in COVID-19. Mol Cell Biochem 476:553-574

71. Padhan K et al (2007) Severe acute respiratory syndrome coronavirus Orf3a protein interacts with caveolin. J Gen Virol 88:3067-3077

72. Hussain A, Das SR, Tanwar C, Jameel S (2007) Oligomerization of the human immunodeficiency virus type 1 (HIV-1) Vpu protein-a genetic, biochemical and biophysical analysis. Virol J $4: 81$

73. Chaudhry A et al (2005) The Nef protein of HIV-1 induces loss of cell surface costimulatory molecules CD80 and CD86 in APCs. J Immunol 175:4566-4574

74. Hussain A, Wesley C, Khalid M, Chaudhry A, Jameel S (2008) Human immunodeficiency virus type $1 \mathrm{Vpu}$ protein interacts with CD74 and modulates major histocompatibility complex class II presentation. J Virol 82:893-902

Publisher's Note Springer Nature remains neutral with regard to jurisdictional claims in published maps and institutional affiliations. 